\title{
Frequency, prognosis and treatment modalities of newly diagnosed small bowel cancer with liver metastases
}

\author{
Xiaorong Ye, Lifu Wang, Yongjun Xing and Chengjun Song ${ }^{*}$
}

\begin{abstract}
Background: Population-based analysis for the liver metastases of small bowel cancer is currently lacking. This study aimed to analyze the frequency, prognosis and treatment modalities for newly diagnosed small bowel cancer patients with liver metastases.

Methods: Patients with small bowel cancer diagnosed from 2010 to 2015 were extracted from the Surveillance, Epidemiology, and End Results (SEER) database. Binary logistic regression analysis was performed to determine predictors for the presence of liver metastases at diagnosis. Kaplan-Meier method and Cox regression analyses were performed for survival analyses.

Results: A total of 1461 small bowel cancer patients with liver metastases at initial diagnosis were identified, representing $16.5 \%$ of the entire set and $63.9 \%$ of the subset with metastatic disease to any distant site. Primary tumor with poorer histological type, larger tumor size, later N staging, more extrahepatic metastatic sites, and tumor on lower part of small intestine had increased propensity of developing liver metastases. The combined diagnostic model exhibited acceptable diagnostic efficiency with AUC value equal to 0.749. Patients with liver metastases had significant poorer survival $(P<0.001)$ than those without liver metastases. In addition, combination of surgery and chemotherapy $(H R=0.27, P<0.001)$ conferred the optimal survival for patients with adenocarcinoma, while the optimal treatment options for NEC and GIST seemed to be surgery alone ( $H R=0.24, P<0.001)$ and chemotherapy alone $(H R=0.08, P=0.022)$, respectively.

Conclusions: The combined predictor had a good ability to predict the presence of liver metastases. In addition, those patients with different histologic types should be treated with distinct therapeutic strategy for obtaining optimal survival.
\end{abstract}

Keywords: Small bowel cancer, Liver metastases, Frequency, Prognosis, Treatment modality

\section{Background}

Small bowel cancer represents a heterogenous group of malignancies, which occurs mainly in the three anatomical segments of the small intestine, including duodenum, jejunum and ileum [1, 2]. Although the most common histological type are adenocarcinoma, carcinoids,

*Correspondence: chengjun_song@163.com

Department of Trauma Surgery, The Lishui People's Hospital, 15 Dazhong

Street, Lishui 323000, Zhejiang, People's Republic of China sarcomas and lymphomas, more than forty different histological subtypes have been described recently [3]. In contrast to large bowel cancer, the incidence of small bowel cancer has been increasing. Based on the latest cancer statistic report, an estimated 10,470 new cases of small bowel cancer are expected to be diagnosed in 2018 in the United States nationally, with 1450 deaths caused by this disease [4]. Although rare, small bowel cancers have an incidence rate comparable to testicular cancer, 
chronic myeloid leukaemia, Hodgkin disease and anal cancer [4].

A certain proportion of small bowel cancer patients presented with evidence of distant metastases at the initial diagnosis, wherein liver exhibited the most common metastatic organ [5, 6]. Evidently, the presence of liver metastases served as an important predictor for worse prognosis of small bowel cancer [6,7], which may due to the increasing tumor burden and impairment of vital organ function caused by disease progression. Owing to the rarity of small bowel cancer with liver metastases, a population-based study regarding to the frequency as well as the prognosis for those population was still lacking. Meanwhile, due to the rarity and non-specific presentation, a major part of patients were diagnosed with advanced stage $[8,9]$, causing a controversial therapeutic strategy, especially for those patients with metastasis disease [10]. Although it is our belief that metastasis cancer (IV stage) is incurable, a few researches had showed that hepatic resection in patients with oligometastatic liver disease might improve survival $[11,12]$. Moreover, palliative surgery also might be necessary in selected cases for relief of bowel obstruction $[13,14]$. Several previous studies have indicated that adjuvant chemotherapy was associated with improved survival for patients with small bowel cancer $[15,16]$. However, other retrospective studies did not demonstrate survival benefit of adjuvant therapy $[5,17]$. Therefore, a large population based study concentrating on describing epidemiologic characteristics, prognosis and optimal treatment modalities of small bowel cancer patients with liver metastases was urgently needed.

In current study, we investigated the incidence and predictors for liver metastases among patients with small bowel cancer by using the SEER database. The prognostic factors associated with the survival of patients with liver metastases were subsequently studied. Furthermore, we also attempted to explore the optimal treatment modalities based on the survival data of small bowel cancer patients with liver metastases.

\section{Methods}

\section{Database and case selection}

Data was obtained from the recently released SEER database [Incidence-SEER 18 Regs Custom Data (with additional treatment fields), Nov 2018 Sub], which collected cancer data that covers about 28 percent of the United States population [4]. We used SEER"Stat software version 8.3.6 (National Cancer Institute, USA) to access the data from SEER database. A total of 13,009 patients with small bowel cancer (Site recode International Classification of Diseases for Oncology-3 (ICD-O-3)/WHO 2008: small intestine) with malignant behavior who were diagnosed from 2010 to 2015 were extracted from the database. Only patients with one primary cancer were included in this study. Moreover, patients with incomplete follow-up, unknown liver metastasis information, and histological type other than adenocarcinoma, neuroendocrine tumors (NETs) and gastrointestinal stromal tumors (GISTs) were excluded. Since it was difficult to accurately differentiate NET or NEC in SEER program based on the recent 2019 WHO classification, NEC and NET were classified into one category as NETs in this study. A total of 8831 eligible small bowel cancer patients were subjected to binary logistic regression analysis to explore the risk factors for the presence of liver metastases. Then, 1461 eligible small bowel cancer patients with liver metastases were selected to perform univariate and multivariate Cox regression analysis for the purpose of exploring the prognostic factors. Furthermore, after exclusion of 2 patients with unknown treatment modalities (surgery, chemotherapy and radiotherapy), we performed multivariate Cox regression analysis to explore the optimal treatment option with greatest survival benefits for small bowel cancer patients who had liver metastases. The flowchart of case selection was shown in Fig. 1.

\section{Covariates}

The analysis involved multiple variables including demographic characteristics (year of diagnosis, age at diagnosis, marital status, insurance status, race, and gender), disease characteristics (primary site, histologic grade, tumor size, liver metastases, AJCC $\mathrm{T}$ and $\mathrm{N}$ stage, and numbers of extrahepatic metastatic sites), and treatment characteristics (surgery, chemotherapy, and radiotherapy). Specially, the continuous variables, including age at diagnosis and tumor size, were transformed into categorical variables. Marital status including single, divorced, widowed, separated and domestic partner were classified into unmarried. Insurance recode including insured and any medicaid were classified into insured. Vital status record recode and cause-specific death classification were utilized to define the main outcomes including overall survival (OS) and cancer-specific survival (CSS).

\section{Statistical analysis}

Descriptive statistic was utilized to summarize the baseline characteristics of patients with liver metastases among the entire set, or among the patients with metastatic disease to any distant site at the time of cancer diagnosis. Clinicopathologic characteristics between patients with or without liver metastases were compared using Pearson chi-square tests. Univariate and multivariable logistic regression were performed to determine predictors of the presence of liver metastases 
Patients with small bowel cancer diagnosed between 2010 and 2015 in SEER database $(n=13009)$

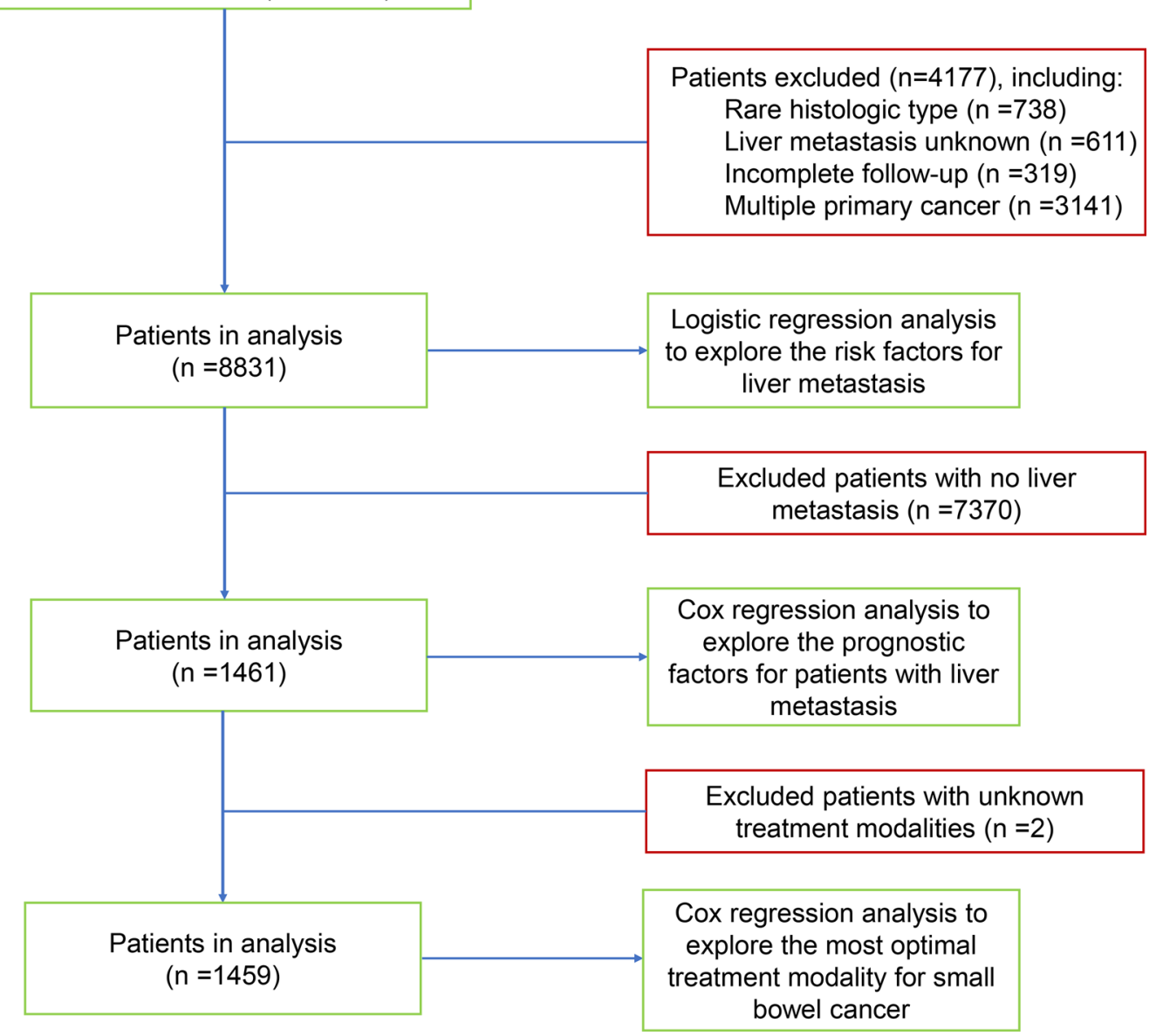

Fig. 1 Flowchart of data selection and grouping. SEER surveillance, epidemiology, and end results program

at diagnosis. Kaplan-Meier plot and log-rank test were used to compare differences of OS and CSS between patients with or without liver metastases. The univariate and multivariate Cox regression analysis were used to estimate hazard ratio (HR) for OS and CSS. In addition, Cox regression model were also conducted to put up multiple comparison across different histological types of small bowel cancer for survival benefit of different treatment modalities by setting up different reference.

Descriptive statistic, Pearson Chi-square test, binary logistic regression model, and Cox proportional hazards model were performed using SPSS 24.0 (IBM Corp). Kaplan-Meier plot and log-rank test were plotted or conducted by using $\mathrm{R}$ software version 3.6.0. A 2-sided $P$ value of $<0.05$ was considered as statistical significance unless otherwise stated.

\section{Results}

Demographic characteristics of patients

Based on the inclusion criteria, a total of 8831 patients with small bowel cancer were extracted from the SEER database, including 2457 (27.8\%) adenocarcinoma, 5406 neuroendocrine tumors (NETs) (61.2\%), and 968 gastrointestinal stromal tumors (GISTs) (11\%). Among the 2285 (25.9\%) patients who had synchronous metastases at the time of diagnosis, a total of 1461 (63.9\%) patients presented with synchronous liver metastases, which consisted of 506 adenocarcinoma, 863 NETs, and 92 GISTs. The detailed demographic and clinical characteristics of those patients with small bowel cancer were summarized in Table 1.

The baseline characteristics between patients with or without liver metastases were compared by Chisquare test (Additional file 1: Table S1). As shown in 
Table 1 Clinical characteristics of small bowel cancer patients with liver metastases at diagnosis

\begin{tabular}{|c|c|c|c|c|c|c|}
\hline \multirow[t]{2}{*}{ Variable } & \multicolumn{3}{|l|}{ Patients, no } & \multicolumn{2}{|c|}{$\begin{array}{l}\text { Proportion of liver metastases, } \\
\%\end{array}$} & \multirow{2}{*}{$\begin{array}{l}\text { Survival among patients } \\
\text { with liver metastases, median } \\
\text { (IQR), mo }\end{array}$} \\
\hline & $\begin{array}{l}\text { With small bowel } \\
\text { cancer }(n=8831)\end{array}$ & $\begin{array}{l}\text { With metastatic } \\
\text { disease } \\
(\mathrm{n}=2285)\end{array}$ & $\begin{array}{l}\text { With liver } \\
\text { metastases } \\
(n=1461)\end{array}$ & $\begin{array}{l}\text { Among } \\
\text { entire } \\
\text { cohort }\end{array}$ & $\begin{array}{l}\text { Among subset } \\
\text { with metastatic } \\
\text { disease }\end{array}$ & \\
\hline \multicolumn{7}{|l|}{ Age } \\
\hline$<40$ & 369 & 86 & 64 & 17.34 & 74.42 & $38.3(18.0-59.0)$ \\
\hline $40-59$ & 2998 & 782 & 509 & 16.98 & 65.09 & $38.7(20.0-57.0)$ \\
\hline $60-79$ & 4371 & 1136 & 727 & 16.63 & 64.00 & $33.5(14.0-51.0)$ \\
\hline$\geq 80$ & 1093 & 281 & 161 & 14.73 & 57.30 & $23.4(2.0-40.0)$ \\
\hline \multicolumn{7}{|l|}{ Race } \\
\hline Black & 1485 & 365 & 241 & 16.23 & 66.03 & $32.8(13.0-51.0)$ \\
\hline White & 6791 & 1806 & 1148 & 16.90 & 63.57 & $34.7(15.0-54.0)$ \\
\hline Others $^{a}$ & 465 & 110 & 69 & 14.84 & 62.73 & $31.7(13.0-49.5)$ \\
\hline Unknown & 90 & 4 & 3 & 3.33 & 75.00 & $32.3(16.0-50.3)$ \\
\hline \multicolumn{7}{|l|}{ Gender } \\
\hline Male & 4553 & 1179 & 765 & 16.80 & 64.89 & $34.0(14.5-52.0)$ \\
\hline Female & 4278 & 1106 & 696 & 16.27 & 62.93 & $34.5(14.0-53.0)$ \\
\hline \multicolumn{7}{|l|}{ Insurance status } \\
\hline No & 263 & 78 & 52 & 19.77 & 66.67 & $35.3(12.0-59.0)$ \\
\hline Yes & 8373 & 2179 & 1390 & 16.60 & 63.79 & $34.2(15.0-53.0)$ \\
\hline Unknown & 195 & 28 & 19 & 9.74 & 67.86 & $33.2(14.0-51.0)$ \\
\hline \multicolumn{7}{|l|}{ Marital status } \\
\hline Unmarried & 3222 & 842 & 529 & 16.42 & 62.83 & $31.5(12.0-50.0)$ \\
\hline Married & 5089 & 1339 & 859 & 16.88 & 64.15 & $36.0(16.0-55.0)$ \\
\hline Unknown & 520 & 104 & 73 & 14.04 & 70.19 & $34.2(16.0-53.8)$ \\
\hline \multicolumn{7}{|l|}{ Primary site } \\
\hline Duodenum & 3201 & 667 & 438 & 13.68 & 65.67 & $28.3(8.0-46.0)$ \\
\hline Jejunum & 848 & 232 & 111 & 13.09 & 47.85 & $35.6(17.0-54.0)$ \\
\hline Ileum & 2572 & 682 & 458 & 17.81 & 67.16 & $39.5(21.0-57.0)$ \\
\hline Other site ${ }^{b}$ & 141 & 27 & 16 & 11.35 & 59.26 & $36.0(16.0-56.5)$ \\
\hline Unknown & 2069 & 677 & 438 & 21.17 & 64.70 & $36.1(17.0-55.0)$ \\
\hline \multicolumn{7}{|l|}{ Grade } \\
\hline I & 3704 & 691 & 464 & 12.53 & 67.15 & $37.9(20.0-55.0)$ \\
\hline$\|$ & 1908 & 483 & 290 & 15.20 & 60.04 & $30.9(13.0-48.0)$ \\
\hline III & 849 & 332 & 186 & 21.91 & 56.02 & $18.5(3.0-27.0)$ \\
\hline IV & 125 & 77 & 27 & 21.60 & 35.06 & $29.9(14.0-46.0)$ \\
\hline Unknown & 2245 & 1514 & 494 & 22.00 & 32.63 & $37.2(14.0-60.5)$ \\
\hline \multicolumn{7}{|l|}{ Histologic type } \\
\hline Adenocarcinoma & 2457 & 886 & 506 & 20.59 & 57.11 & $20.0(3.0-29.0)$ \\
\hline NEC & 5406 & 1205 & 863 & 15.96 & 71.62 & $39.5(21.0-57.0)$ \\
\hline GISS & 968 & 194 & 92 & 9.50 & 47.42 & $41.1(21.0-60.0)$ \\
\hline \multicolumn{7}{|l|}{ Tstage } \\
\hline $\mathrm{T} 1$ & 1382 & 157 & 98 & 7.09 & 62.42 & $33.2(14.0-52.0)$ \\
\hline $\mathrm{T} 2$ & 1306 & 157 & 114 & 8.73 & 72.61 & $40.8(22.0-60.0)$ \\
\hline T3 & 2767 & 627 & 418 & 15.11 & 66.67 & $38.4(19.0-57.0)$ \\
\hline T4 & 2128 & 803 & 427 & 20.07 & 53.18 & $29.7(11.0-46.0)$ \\
\hline Unknown & 1248 & 541 & 404 & 32.37 & 74.68 & $27.1(4.25-46.0)$ \\
\hline \multicolumn{7}{|l|}{ Tumor size, cm } \\
\hline $0-1$ & 1518 & 86 & 52 & 3.43 & 60.47 & $38.3(20.0-56.0)$ \\
\hline $1-2$ & 1897 & 413 & 267 & 14.07 & 64.65 & $38.9(20.0-57.0)$ \\
\hline
\end{tabular}


Table 1 (continued)

\begin{tabular}{|c|c|c|c|c|c|c|}
\hline \multirow[t]{2}{*}{ Variable } & \multicolumn{3}{|l|}{ Patients, no } & \multicolumn{2}{|c|}{$\begin{array}{l}\text { Proportion of liver metastases, } \\
\%\end{array}$} & \multirow{2}{*}{$\begin{array}{l}\text { Survival among patients } \\
\text { with liver metastases, median } \\
\text { (IQR), mo }\end{array}$} \\
\hline & $\begin{array}{l}\text { With small bowel } \\
\text { cancer }(n=8831)\end{array}$ & $\begin{array}{l}\text { With metastatic } \\
\text { disease } \\
(n=2285)\end{array}$ & $\begin{array}{l}\text { With liver } \\
\text { metastases } \\
(n=1461)\end{array}$ & $\begin{array}{l}\text { Among } \\
\text { entire } \\
\text { cohort }\end{array}$ & $\begin{array}{l}\text { Among subset } \\
\text { with metastatic } \\
\text { disease }\end{array}$ & \\
\hline $2-5$ & 2523 & 751 & 488 & 19.34 & 64.98 & $34.7(15.0-53.0)$ \\
\hline$>5$ & 1325 & 347 & 176 & 13.28 & 50.72 & $33.8(14.0-52.0)$ \\
\hline Unknown & 1568 & 688 & 478 & 30.48 & 69.48 & $24.3(16.0-42.0)$ \\
\hline \multicolumn{7}{|l|}{ N stage } \\
\hline No & 4732 & 859 & 531 & 11.22 & 61.82 & $34.5(14.0-54.0)$ \\
\hline $\mathrm{N} 1$ & 3430 & 1096 & 728 & 21.22 & 66.42 & $36.7(18.0-55.0)$ \\
\hline N2 & 315 & 115 & 51 & 16.19 & 44.35 & $20.9(7.0-29.0)$ \\
\hline Unknown & 354 & 215 & 151 & 42.66 & 70.23 & $18.9(2.0-29.0)$ \\
\hline \multicolumn{7}{|l|}{ M stage } \\
\hline MO & 6546 & 6546 & 0 & 0.00 & 0.00 & $37.1(18.0-56.0)$ \\
\hline M1 & 2285 & 2285 & 1461 & 63.94 & 63.94 & $26.1(6.0-42.0)$ \\
\hline \multicolumn{7}{|c|}{ Extrahepatic metastatic sites to bone, lung, and brain, No } \\
\hline 0 & 8488 & 1953 & 1255 & 14.79 & 64.26 & $35.0(15.0-54.0)$ \\
\hline 1 & 243 & 243 & 142 & 58.44 & 58.44 & $14.1(2.0-21.0)$ \\
\hline 2 & 16 & 16 & 9 & 56.30 & 56.30 & $9.5(2.3-14.8)$ \\
\hline Unknown & 84 & 73 & 55 & 65.48 & 75.34 & $20.6(1.3-36.8)$ \\
\hline \multicolumn{7}{|l|}{ Surgery } \\
\hline No & 1865 & 946 & 674 & 36.14 & 71.24 & $19.7(3.0-30.0)$ \\
\hline Yes & 6935 & 1335 & 785 & 11.32 & 58.80 & $38.1(19.0-56.0)$ \\
\hline Unknown & 31 & 4 & 2 & 6.45 & 50.00 & $36.7(14.0-56.0)$ \\
\hline \multicolumn{7}{|c|}{ Radiotherapy } \\
\hline No & 8563 & 2177 & 1387 & 16.20 & 63.71 & $34.5(15.0-53.0)$ \\
\hline Yes & 268 & 108 & 74 & 27.61 & 68.52 & $24.3(9.0-37.8)$ \\
\hline \multicolumn{7}{|c|}{ Chemotherapy } \\
\hline No & 6820 & 1406 & 918 & 13.46 & 65.29 & $35.6(16.0-55.0)$ \\
\hline Yes & 2011 & 879 & 543 & 27.00 & 61.77 & $29.7(12.0-44.0)$ \\
\hline
\end{tabular}

IQR interquartile range, $C I$ confidence interval, NEC neuroendocrine carcinoma, GISS gastrointestinal stromal sarcoma

a Asian and American Indians

b Meckels diverticulum, and overlapping lesion of small intestine

Additional file 1: Table S1, a significant difference could be found in constituent ratio of race, insurance status, tumor primary site, grade, histological type, AJCC T and $\mathrm{N}$ stage, tumor size, number of extrahepatic metastatic sites, surgery, radiotherapy, and chemotherapy. Patients without liver metastases had higher proportion of duodenum (37.5\% vs $30.0 \%$ ), jejunum tumor ( $10.0 \%$ vs $7.6 \%)$, but less ileum tumor $(28.7 \%$ vs $31.3 \%)$ as compared with patients diagnosed with liver metastases. Patients who had liver metastases tended to have higher histological grade, later AJCC $\mathrm{T}$ and $\mathrm{N}$ stage, and more numbers of extrahepatic metastatic sites than patients without liver metastases. In addition, patients with liver metastasis had received more adjuvant therapy, such as chemotherapy $(37.2 \%$ vs $19.9 \%)$ or radiotherapy (5.1\% vs $2.6 \%$ ), but less surgical treatment (53.7\% vs $83.4 \%)$ than patients without liver metastases.

\section{Predictors for the presence of liver metastases}

In order to identify the possible predictors associated with occurrence of liver metastases, univariate and multivariate binary logistic regression analysis were performed (Table 2). The multivariate analysis showed that jejunum (vs duodenum; $\mathrm{OR}=1.38 ; 95 \% \mathrm{CI}[1.07-1.78] ; P=0.012$ ), ileum (vs duodenum; OR $=2.06 ; 95 \%$ CI $[1.70-2.50]$; $P<0.001$ ), grade III (vs grade I; OR $=1.54 ; 95 \%$ CI $[1.21-$ 1.96 ]; $P=0.001$ ), grade IV (vs grade I; OR $=2.54 ; 95 \% \mathrm{CI}$ [1.56-4.12]; $P<0.001$ ), tumor size $1-2 \mathrm{~cm}$ (vs tumor size $0-1 \mathrm{~cm} ; \mathrm{OR}=3.83 ; 95 \% \mathrm{CI}[2.66-5.52] ; P<0.001)$, tumor size $2-5 \mathrm{~cm}$ (vs tumor size $0-1 \mathrm{~cm}$; $\mathrm{OR}=5.53$; $95 \% \mathrm{CI}$ 
Table 2 Factors associated with the presence of liver metastases at diagnosis of small bowel cancer

\begin{tabular}{|c|c|c|c|c|}
\hline \multirow[t]{2}{*}{ Variables } & \multicolumn{2}{|c|}{$\begin{array}{l}\text { Univariate logistic } \\
\text { model }\end{array}$} & \multicolumn{2}{|c|}{$\begin{array}{l}\text { Multivariate logistic } \\
\text { model }\end{array}$} \\
\hline & OR $(95 \% \mathrm{Cl})$ & $P$ value & OR $(95 \% \mathrm{Cl})$ & $P$ value \\
\hline
\end{tabular}

Age$$
<40
$$

40-59

60-79

$\geq 80$

Race

\section{Black}

White

Others $^{a}$

Unknown

Gender

Male

Female

Insurance status

No

Yes

Unknown

Marital status

Unmarried

Married

Unknown

Primary site

Duodenum

Jejunum

lleum

Other site $e^{b}$

Unknown

Grade

I

॥

III

IV

Unknown

Histologic type

Adenocarcinoma

NEC

GISS

T stage

T1

T2

T3

T4

Unknown

Tumor size, cm

$0-1$
$1-2$
$2-5$

Reference

0.98 (0.73-1.30)

$0.95(0.72-1.26)$

$0.82(0.60-1.13)$

Reference

1.05 (0.90-1.22)

$0.90(0.67-1.20)$

$0.18(0.06-0.57)$

Reference

0.96 (0.86-1.08)

Reference

0.81 (0.59-1.10)

$0.44(0.25-0.77)$

Reference

1.03 (0.92-1.16)

$0.83(0.64-1.08)$

Reference

$0.95(0.76-1.19)$

$1.37(1.19-1.58)$

$0.81(0.48-1.37)$

$1.69(1.46-1.96)$

Reference

1.25 (1.07-1.47)

$1.96(1.62-2.37)$

$1.92(1.24-2.98)$

$1.97(1.71-2.27)$

Reference

$0.73(0.65-0.83)$

$0.41(0.32-0.51)$

Reference

1.25 (0.95-1.66)

$2.33(0.95-1.66)$

$3.29(1.85-2.94)$

$6.27(4.95-7.95)$

Reference

4.62 (3.40-6.27)

$6.76(5.04-9.07)$

NA
0.860
0.725
0.229

NA

0.528

0.474

0.004

NA

0.501

$$
\text { NA }
$$

0.175

0.004

NA

0.583

0.171

Reference

$0.6541 .38(1.07-1.78) \quad 0.012$

$<0.0012 .06(1.70-2.50)<0.001$

$0.4291 .18(0.66-2.09) \quad 0.580$

$<0.0012 .28(1.89-2.74)<0.001$

Reference

$0.0051 .11(0.92-1.34) \quad 0.263$

$<0.0011 .54(1.21-1.96) \quad 0.001$

$0.0032 .54(1.56-4.12)<0.001$

$<0.0011 .69(1.43-2.00)<0.001$

Reference

$<0.0010 .98(0.80-1.18) \quad 0.800$

$<0.0010 .56(0.41-0.75)<0.001$

Reference

$0.1160 .57(0.40-0.80) \quad 0.001$

$<0.0010 .71(0.52-0.97) \quad 0.029$

$<0.0010 .94(0.69-1.28) \quad 0.679$

$<0.0011 .62(1.19-2.21) \quad 0.002$

Reference

$<0.0013 .83(2.66-5.52)<0.001$ $<0.0015 .53(3.85-7.94)<0.001$
Table 2 (continued)

\begin{tabular}{|c|c|c|c|c|}
\hline \multirow[t]{2}{*}{ Variables } & \multicolumn{2}{|c|}{$\begin{array}{l}\text { Univariate logistic } \\
\text { model }\end{array}$} & \multicolumn{2}{|c|}{$\begin{array}{l}\text { Multivariate logistic } \\
\text { model }\end{array}$} \\
\hline & OR $(95 \% \mathrm{Cl})$ & $P$ value & OR $(95 \% \mathrm{CI})$ & $P$ value \\
\hline$>5$ & $4.32(3.14-5.94)$ & $<0.001$ & $4.38(2.91-6.59)$ & $<0.001$ \\
\hline Unknown & $12.4(9.19-16.6)$ & $<0.001$ & $6.75(4.67-9.73)$ & $<0.001$ \\
\hline \multicolumn{5}{|l|}{ N stage } \\
\hline NO & Reference & & Reference & \\
\hline N1 & $2.13(1.89-2.41)$ & $<0.001$ & $1.87(1.60-2.18)$ & $<0.001$ \\
\hline N2 & $1.53(1.12-2.09)$ & 0.008 & $1.19(0.84-1.69)$ & 0.339 \\
\hline Unknown & $5.89(4.68-7.40)$ & $<0.001$ & $2.12(1.62-2.77)$ & $<0.001$ \\
\hline \multicolumn{5}{|c|}{ Extrahepatic metastatic sites to bone, lung, and brain, No } \\
\hline 0 & Reference & & Reference & \\
\hline 1 & $8.10(6.24-10.5)$ & $<0.001$ & $5.14(3.86-6.84)$ & $<0.001$ \\
\hline 2 & $7.41(2.76-19.9)$ & $<0.001$ & $4.35(1.53-12.3)$ & 0.006 \\
\hline Unknown & $10.9(6.94-17.2)$ & $<0.001$ & $6.29(3.89-10.2)$ & $<0.001$ \\
\hline
\end{tabular}

Cl confidence interval, OR odds ratio, NEC neuroendocrine carcinoma, GISS gastrointestinal stromal sarcoma

a Asian and American Indians

b Meckels diverticulum, and overlapping lesion of small intestine

[3.85-7.94]; $P<0.001$ ), tumor size $>5 \mathrm{~cm}$ (vs tumor size $0-1 \mathrm{~cm} ; \mathrm{OR}=4.38 ; 95 \% \mathrm{CI}$ [2.91-6.59]; $P<0.001$ ), N1 (vs N0; OR $=1.87 ; 95 \%$ CI $[1.60-2.18] ; P<0.001), 1$ extrahepatic metastatic site (vs 0 extrahepatic metastatic site; $\mathrm{OR}=5.14 ; 95 \%$ CI $[3.86-6.84] ; P<0.001), 2$ extrahepatic metastatic site (vs 0 extrahepatic metastatic site; $\mathrm{OR}=4.35$; 95\% CI [1.53-12.3]; $P=0.006)$ were significantly associated with greater odds of having liver metastases at initial diagnosis. On the contrary, gastrointestinal stromal tumor (GIST) (vs adenocarcinoma; $\mathrm{OR}=0.56$; 95\% CI [0.41-0.75]; $P<0.001)$ were significantly associated with lower odds of liver metastases at diagnosis. Taken together, these data suggested that small bowel cancer patients with factors like lower part of small intestine, poorer histological grade, larger tumor size, later $\mathrm{N}$ staging, and presence of more extrahepatic metastatic sites showed an increased propensity for developing liver metastases.

Subsequently, the ROC curve was plotted to evaluate the predicting performance (Fig. 2). The multivariable logistic model, which incorporated seven significant variables, exhibited dramatically higher AUC (AUC: 0.749; 95\% CI [0.735-0.762]; $P<0.001)$ value than separate variables, including primary site (AUC: 0.557 ; 95\% CI [0.5410.574]), grade (AUC: 0.580; 95\% CI [0.564-0.596]), histologic type (AUC: $0.443 ; 95 \%$ CI [0.428-0.459]), AJCC T stage (AUC: 0.653; 95\% CI [0.638-0.668]), tumor size (AUC: 0.643 ; 95\% CI [0.628-0.657]), AJCC N stage (AUC: 0.614; 95\% CI [0.598-0.630]), and number of extrahepatic metastatic sites (AUC: $0.561 ; 95 \%$ CI 


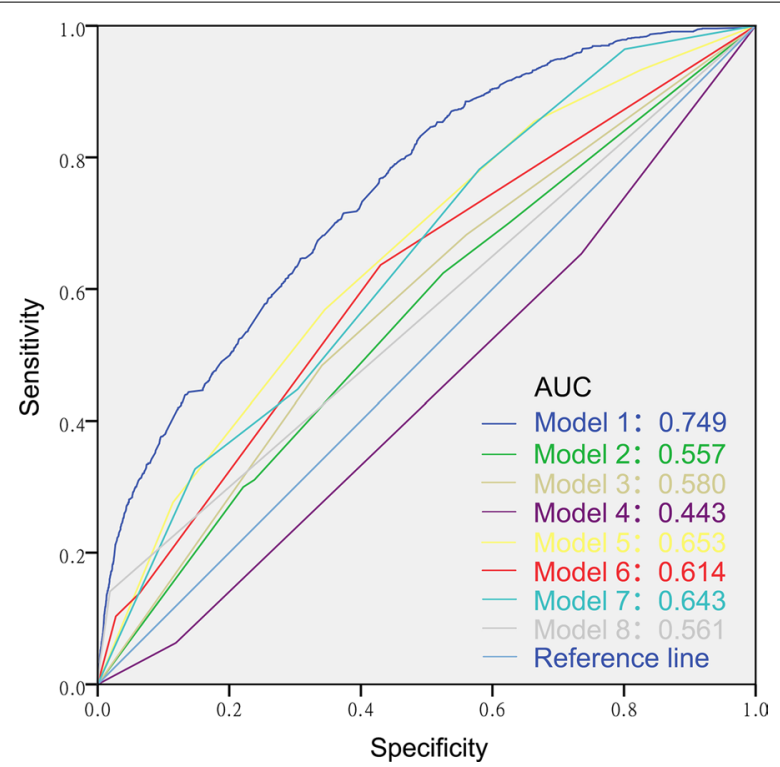

Fig. 2 ROC curves to predict the presence of liver metastases in the entire set stratified by model 1 (combined predictors, AUC: 0.749), model 2 (primary site, AUC: 0.557), model 3 (grade, AUC: 0.580), model 4 (histologic type, AUC: 0.443), model 5 (AJCC T stage, AUC: 0.653), model 6 (AJCC N stage, AUC: 0.614), model 7 (tumor size, AUC: 0.643 ) and model 8 (number of extrahepatic metastatic sites, AUC: $0.561)$

[0.544-0.578]). These results suggested that our model had a good performance for discriminating patients prone to occur liver metastases.

\section{Survival analysis of small bowl cancer patients with liver metastases}

The Kaplan-Meier method was used to compare the OS and CSS between patients with or without liver metastases. As shown in Fig. 3a, b, patients with liver metastases had significantly poorer OS $(P<0.001)$ and CSS $(P<0.001)$ than those without liver metastases in the total set. Similar trends were also seen in all subsets, including adenocarcinoma, NETs and GISTs patients (Fig. 3c-h).

Of all the 1461 patients who had liver metastases, a total of $709(48.5 \%)$ patients were dead at the time of last follow-up, among which 660 (45.2\%) patients were dead directly from small bowel cancer. Subsequently, among the subsets (adenocarcinoma, NETs and GISTs) with liver metastases, univariate and multivariate Cox regression analyses were separately performed to identify factors which significantly associated with OS and CSS. Additional file 2: Table S2, Additional file 3: Table S3, Additional file 4: Table S4 showed univariate analysis for OS and CSS among patients with adenocarcinoma, NETs and GISTs, respectively. The multivariable Cox analysis for adenocarcinoma set showed that tumors occurred in jejunum (vs. duodenum) was significantly associated with an increased $\mathrm{OS}(\mathrm{HR}=0.65 ; 95 \% \mathrm{CI}$ $[0.46-0.92] ; P=0.014)$ and $\mathrm{CSS}(\mathrm{HR}=0.61 ; 95 \% \mathrm{CI}$ [0.43-0.88]; $P=0.008$ ) (Table 3). Moreover, as shown in Table 4, the multivariate analysis for NETs indicated that patients with age at $60-79$ (vs age $<40 ; \mathrm{HR}=6.86$; 95\% CI [2.18-21.6]; $P=0.001$ ), $\geq 80$ (vs age $<40 ; \mathrm{HR}=10.1$; 95\% CI [3.09-33.3]; $P<0.001$ ), grade III (vs grade I; $\mathrm{HR}=5.73 ; 95 \%$ CI $[3.22-10.2] ; P<0.001)$, grade IV (vs grade $\mathrm{I} ; \mathrm{HR}=8.46 ; 95 \% \mathrm{CI}[3.81-18.8] ; P<0.001$ ), or 1 extrahepatic metastatic site (vs 0 extrahepatic metastatic site; HR $=2.07 ; 95 \%$ CI $[1.36-3.15] ; P=0.001)$ were significantly associated with decreased OS. On the contrary, patients who had tumors occurred in jejunum (vs duodenum; $\mathrm{HR}=0.39 ; 95 \% \mathrm{CI}$ [0.17-0.91]; $P=0.030$ ), or ileum (vs duodenum; $\mathrm{HR}=0.43 ; 95 \% \mathrm{CI}$ [0.28-0.67]; $P<0.001)$ were significantly associated with increased OS. Similar result was also presented for CSS in Table 4. However, no significant prognostic factor was found among GIST patients who had liver metastases (Additional file 4: Table S4).

\section{Associations of treatment modality and survival outcomes} In order to better understand the survival benefit of various treatment modalities, the prognosis of small bowel cancer patients with liver metastases who had received different treatment modalities were compared (Fig. 4). In total set, patients who received surgery $(P<0.001)$ or combination of surgery and chemotherapy $(P<0.001)$ had significantly favorable prognosis as compared with those who received no treatment (Fig. 4a). However, it seems that patients could not benefit from chemotherapy alone (Fig. 4a). For the adenocarcinoma set, all of the treatment modalities significantly increased patients' CSS $(P<0.05)$ (Fig. 4b). For the NETs set, similar result was obtained to the total set (Fig. 4c). However, for the GISTs set, only chemotherapy alone significantly increased the survival rate as compared with no treatment (Fig. 4d).

Subsequently, multivariate Cox analysis was utilized to unveil the optimal treatment modality for small bowel cancer patients with different histological type. As shown in Table 5, patients with adenocarcinoma could benefit from chemotherapy alone $(\mathrm{HR}=0.35,95 \%$ CI $[0.27-$ $0.44], P<0.001)$ or surgery plus chemotherapy $(\mathrm{HR}=0.27$, 95\% CI [0.18-0.42], $P<0.001)$ when compared with no treatment. By setting different reference, surgery plus chemotherapy seemed to be the best therapeutic option (Surgery \& chemotherapy vs surgery: $\mathrm{HR}=0.37,95 \%$ CI [0.22-0.65], $P<0.001$; Surgery \& chemotherapy vs chemotherapy: $\mathrm{HR}=0.67,95 \% \mathrm{CI}[0.42-1.06], P=0.089$ ). For patients with NETs, treatment modalities including surgery $(\mathrm{HR}=0.24,95 \% \mathrm{CI}[0.14-0.43], P<0.001)$ and surgery \& chemotherapy $(\mathrm{HR}=0.39,95 \%$ CI [0.20-0.73], 


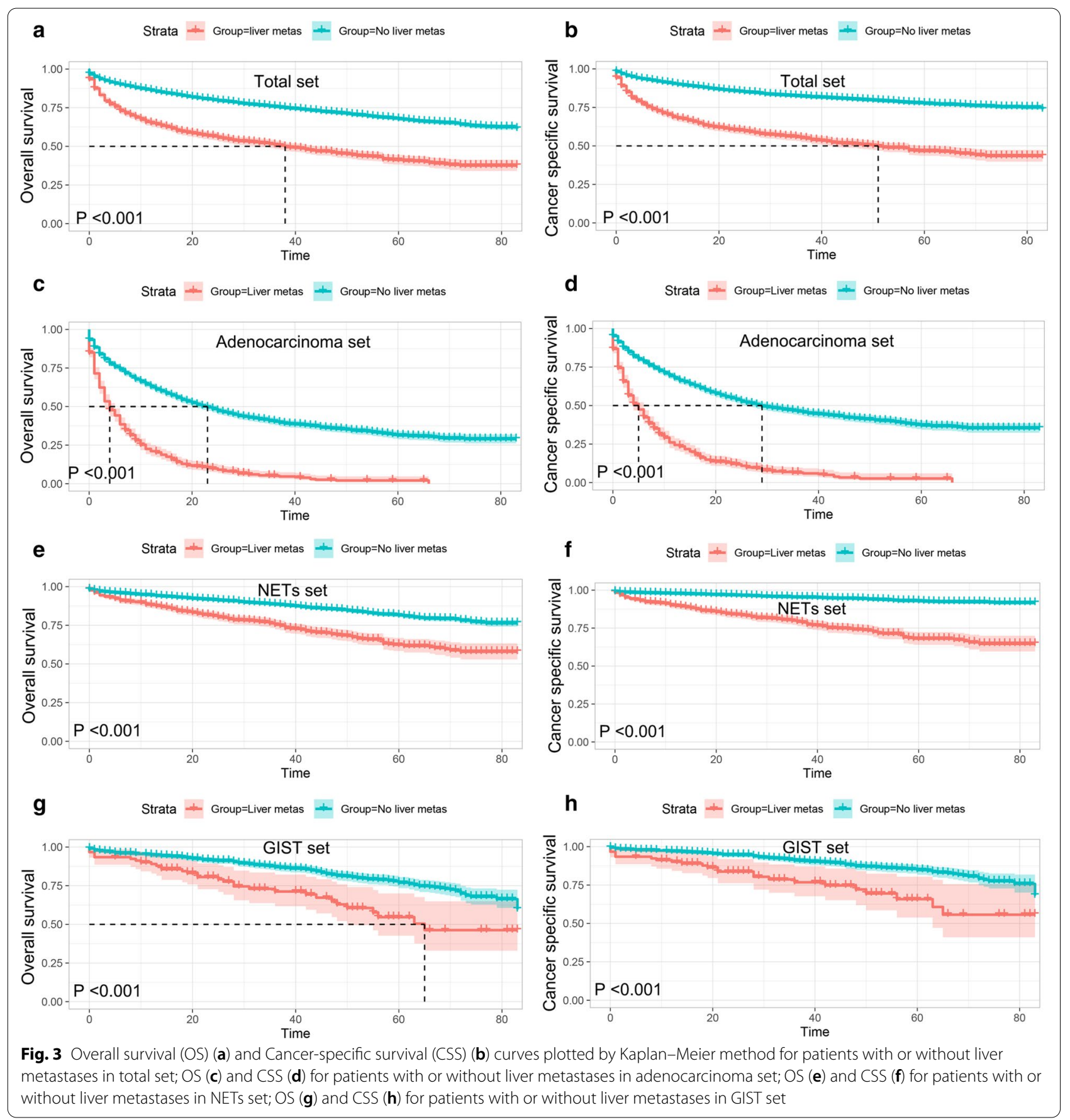

$P=0.004)$ could provide survival benefit when compared with no treatment, and surgery only seemed to be the best therapeutic option (Surgery \& chemotherapy vs surgery: $\quad H R=1.48,95 \%$ CI $[0.91-2.41], \quad P=0.112$; chemotherapy vs surgery: $\mathrm{HR}=2.81,95 \% \mathrm{CI}$ [1.41-5.63], $P=0.003$ ) (Table 5). Moreover, patients with GISTs could only benefit from chemotherapy alone $(\mathrm{HR}=0.08,95 \%$ CI [0.01-0.69], $P=0.022$ ) (Table 5).

\section{Discussion}

The current study described the frequency and prognosis of small bowel cancer patients with liver metastases at their initial diagnosis by using available data from the SEER database. We also explored the predictive indicators for the presence of liver metastases, and sought its optimal treatment modalities based on the survival data, with an attempt to better understand the clinical 
Table 3 Multivariate analysis for overall survival (OS) and cancer-specific survival (CSS) among patients with small bowel adenocarcinoma who had liver metastasis

\begin{tabular}{|c|c|c|c|c|}
\hline \multirow[t]{2}{*}{ Variables } & \multicolumn{2}{|l|}{ OS } & \multicolumn{2}{|l|}{ CSS } \\
\hline & HR $(95 \% \mathrm{Cl})$ & $P$ value & $\mathrm{HR}(95 \% \mathrm{Cl})$ & $P$ value \\
\hline \multicolumn{5}{|l|}{ Age } \\
\hline$<40$ & Reference & & Reference & \\
\hline $40-59$ & $0.81(0.48-1.36)$ & 0.417 & $0.77(0.45-1.29)$ & 0.312 \\
\hline $60-79$ & $0.95(0.58-1.58)$ & 0.854 & $0.83(0.50-1.37)$ & 0.461 \\
\hline$\geq 80$ & $1.52(0.89-2.62)$ & 0.129 & $1.39(0.80-2.40)$ & 0.241 \\
\hline \multicolumn{5}{|l|}{ Race } \\
\hline Black & Reference & & Reference & \\
\hline White & $1.14(0.90-1.45)$ & 0.262 & $1.15(0.90-1.48)$ & 0.255 \\
\hline Others $^{\mathrm{a}}$ & $1.00(0.66-1.52)$ & 0.988 & $1.09(0.71-1.67)$ & 0.687 \\
\hline \multicolumn{5}{|l|}{ Primary site } \\
\hline Duodenum & Reference & & Reference & \\
\hline Jejunum & $0.65(0.46-0.92)$ & 0.014 & $0.61(0.43-0.88)$ & 0.008 \\
\hline Ileum & $0.81(0.55-1.21)$ & 0.307 & $0.86(0.57-1.28)$ & 0.453 \\
\hline Other site ${ }^{b}$ & $0.42(0.15-1.15)$ & 0.090 & $0.46(0.17-1.27)$ & 0.133 \\
\hline Unknown & $0.96(0.72-1.27)$ & 0.774 & $0.97(0.72-1.29)$ & 0.810 \\
\hline \multicolumn{5}{|l|}{ Tstage } \\
\hline T1 & Reference & & Reference & \\
\hline T2 & $1.36(0.62-2.98)$ & 0.445 & $1.52(0.69-3.35)$ & 0.299 \\
\hline T3 & $0.77(0.53-1.11)$ & 0.165 & $0.75(0.51-1.11)$ & 0.155 \\
\hline T4 & $1.02(0.76-1.37)$ & 0.888 & $1.02(0.75-1.38)$ & 0.913 \\
\hline Unknown & $1.30(0.99-1.70)$ & 0.061 & $1.37(1.03-1.82)$ & 0.031 \\
\hline \multicolumn{5}{|l|}{ N stage } \\
\hline NO & Reference & & Reference & \\
\hline N1 & $0.93(0.74-1.16)$ & 0.516 & $0.94(0.75-1.19)$ & 0.600 \\
\hline N2 & $0.93(0.66-1.32)$ & 0.686 & $0.98(0.68-1.40)$ & 0.895 \\
\hline Unknown & $0.96(0.73-1.27)$ & 0.793 & $0.93(0.69-1.24)$ & 0.604 \\
\hline
\end{tabular}

$\mathrm{Cl}$ confidence interval, $H R$ hazard ratio

a Asian and American Indians

b Meckels diverticulum, and overlapping lesion of small intestine

significance of liver metastases. Since early diagnosis and reasonable treatment may improve overall survival and quality of life, it is of great significance to investigate small bowel cancer patients who had liver metastases in a large independent cohort.

In our study, we totally identified 8831 small bowel cancer patients, of which neuroendocrine tumors (61.2\%) were the most common histologic type. This data was not consistent with previous studies that considered adenocarcinomas as the most commonly occurring malignant neoplasms in the small bowel [1, 18]. However, based on the data of small bowel malignancies from National Cancer Data Base (NCDB, 1985-2005), the proportion of carcinoid tumors, which
Table 4 Multivariate analysis for overall survival (OS) and cancer-specific survival (CSS) among patients with small bowel neuroendocrine tumors (NETs) who had liver metastasis

\begin{tabular}{|c|c|c|c|c|}
\hline \multirow[t]{2}{*}{ Variables } & \multicolumn{2}{|l|}{ os } & \multicolumn{2}{|l|}{ CSS } \\
\hline & $\mathrm{HR}(95 \% \mathrm{Cl})$ & $P$ value & $\mathrm{HR}(95 \% \mathrm{Cl})$ & $P$ value \\
\hline \multicolumn{5}{|l|}{ Age } \\
\hline$<40$ & Reference & & Reference & \\
\hline $40-59$ & $2.96(0.93-9.47)$ & 0.067 & $3.71(0.90-15.3)$ & 0.070 \\
\hline $60-79$ & $6.86(2.18-21.6)$ & 0.001 & $8.70(2.14-35.3)$ & 0.002 \\
\hline$\geq 80$ & $10.1(3.09-33.3)$ & $<0.001$ & $11.8(2.78-50.2)$ & 0.001 \\
\hline \multicolumn{5}{|l|}{ Marital status } \\
\hline Unmarried & Reference & & Reference & \\
\hline Married & $0.82(0.63-1.08)$ & 0.153 & $0.82(0.61-1.11)$ & 0.203 \\
\hline Unknown & $0.81(0.45-1.47)$ & 0.490 & $0.83(0.44-1.59)$ & 0.574 \\
\hline \multicolumn{5}{|l|}{ Primary site } \\
\hline Duodenum & Reference & & Reference & \\
\hline Jejunum & $0.39(0.17-0.91)$ & 0.030 & $0.37(0.15-0.92)$ & 0.032 \\
\hline Ileum & $0.43(0.28-0.67)$ & $<0.001$ & $0.37(0.23-0.59)$ & $<0.001$ \\
\hline Other site ${ }^{a}$ & $0.47(0.11-2.00)$ & 0.309 & $0.26(0.04-1.95)$ & 0.191 \\
\hline Unknown & $0.54(0.35-0.82)$ & 0.004 & $0.47(0.30-0.73)$ & 0.001 \\
\hline \multicolumn{5}{|l|}{ Grade } \\
\hline । & Reference & & Reference & \\
\hline$\|$ & $1.14(0.75-1.74)$ & 0.540 & $1.13(0.70-1.82)$ & 0.621 \\
\hline III & $5.73(3.22-10.2)$ & $<0.001$ & $5.32(2.81-10.1)$ & $<0.001$ \\
\hline IV & $8.46(3.81-18.8)$ & $<0.001$ & $10.1(4.50-22.8)$ & $<0.001$ \\
\hline Unknown & $1.98(1.46-2.70)$ & $<0.001$ & $1.97(1.40-2.78)$ & $<0.001$ \\
\hline \multicolumn{5}{|l|}{ N stage } \\
\hline No & Reference & & Reference & \\
\hline N1 & $0.76(0.57-1.02)$ & 0.066 & $0.76(0.55-1.05)$ & 0.098 \\
\hline N2 & $0.78(0.57-1.06)$ & 0.107 & $0.73(0.52-1.03)$ & 0.070 \\
\hline Unknown & $1.20(0.79-1.83)$ & 0.382 & $1.31(0.84-2.05)$ & 0.235 \\
\hline
\end{tabular}

Extrahepatic metastatic sites to bone, lung, and brain, No

\begin{tabular}{llllr}
0 & Reference & & Reference & \\
1 & $2.07(1.36-3.15)$ & 0.001 & $2.32(1.49-3.61)$ & $<0.001$ \\
2 & $1.13(0.73-1.74)$ & 0.590 & $0.99(0.39-2.42)$ & 0.961 \\
Unknown & $0.63(0.27-1.46)$ & 0.283 & $0.52(0.30-1.84)$ & 0.743 \\
\hline
\end{tabular}

$\mathrm{Cl}$ confidence interval, $\mathrm{HR}$ hazard ratio

${ }^{a}$ Meckels diverticulum, and overlapping lesion of small intestine

consists mainly of NET and NEC, increased significantly from $27.5 \%$ to $44.3 \%$, whereas the proportion of adenocarcinomas decreased from $42.1 \%$ to $32.6 \%$ [19]. It was also reported that carcinoid tumors surpassed adenocarcinomas as the most common small bowel tumor [19]. In addition, the incidence of carcinoid tumors increased from 2.1 to 9.3 per million (percent change: $340.5 \%$; annual percentage change: $3.6 \%$ ) from 1973 to 2004, whereas the incidence of adenocarcinoma increased with less pronounced. Despite the existence of potential selection bias, we hold the opinion that the 


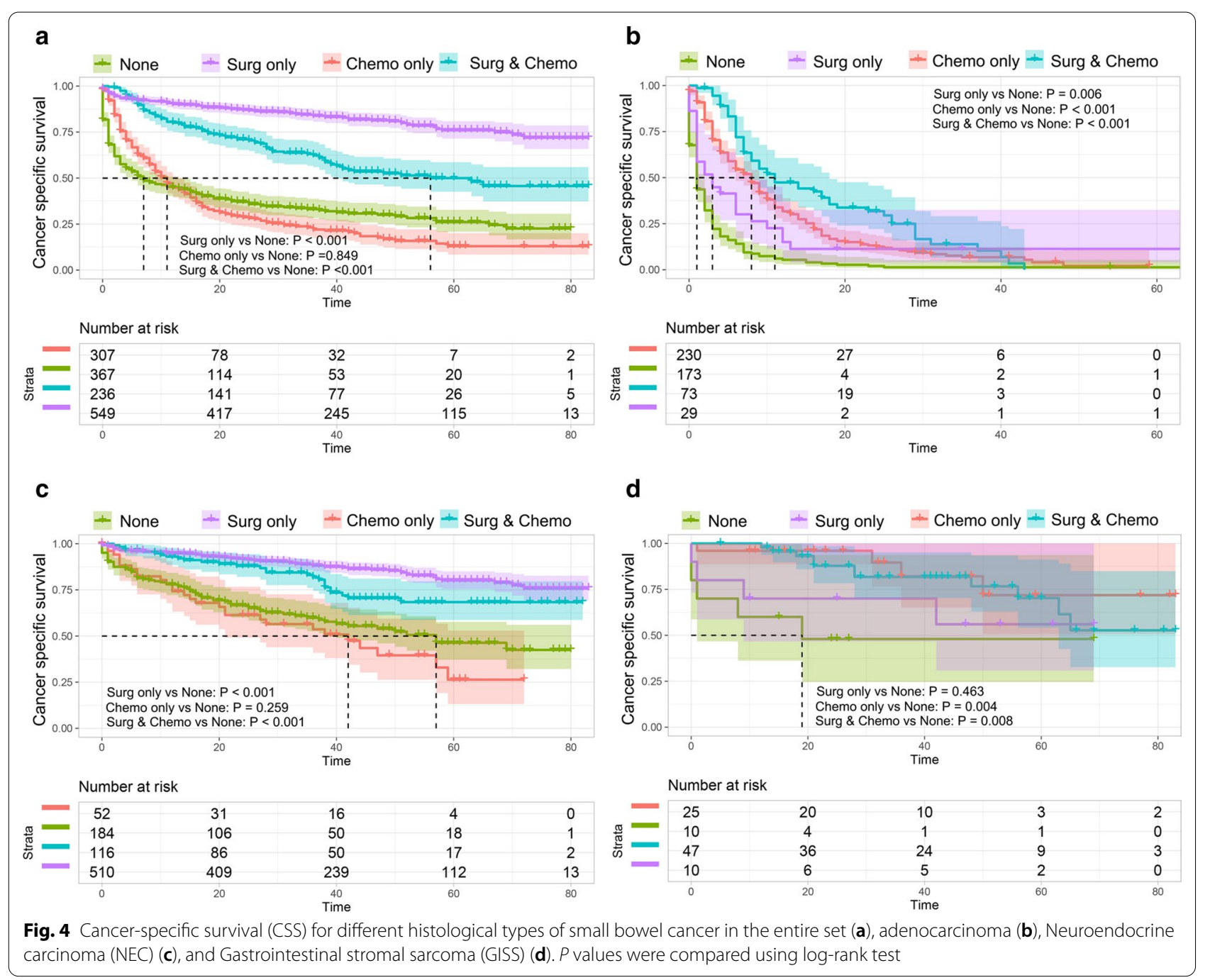

proportion of neuroendocrine carcinomas would have increased recently. We also found that $25.9 \%$ of patients were diagnosed with synchronous metastatic disease among which $63.9 \%$ initially presented with liver metastases. Specifically, distant metastases were occurred in $20.6 \%$ of patients with adenocarcinoma, $16.0 \%$ of NETs, and $9.5 \%$ of GISTs. These result differed slightly from the previous published studies wherein distant metastases were noted on presentation in $24.0 \%$ of adenocarcinoma, $15.6 \%$ of NETs, and $9.5 \%$ of NETs [19].

The risk factors for the occurrence of liver metastases at initial diagnosis were identified using multivariate logistic regression in order to distinguish patients at increased risk for liver metastases. We found that patients were easier to have liver metastases when they had risk factors as follow: tumor located in the lower part of small bowel, poorer histological grade, larger tumor size, later $\mathrm{N}$ staging, and presence of more extrahepatic metastatic sites. However, our study failed to demonstrate that tumors with $\mathrm{T} 4$ and
N2 stage had higher risk of occurring liver metastases in comparison with $\mathrm{T} 0$ and N0, respectively. A similar result was also shown in gastric cancer [20]. This result, to some extent, implied that traditional AJCC TNM staging was not sufficient to predict the presence of liver metastases. As a replacement, our study indicated that the tumor size could effectively predict the occurrence of liver metastases, largely because of the reason that large tumor had more chance to occur lymphatic dissemination, hematogenous dissemination, and serosal invasion. In addition, our results also demonstrated that demographic characteristics, such as age, race and insurance status, were not risk factors for the liver metastases, which was not in accordance with previous studies concentrating on gastric cancer [20-22]. Moreover, based on the multivariate logistic regression model, ROC curves incorporating seven independent risk factors showed the best predictive value, with an AUC value equal to 0.75 , which were significantly higher than single predictors ranging from 0.44 to 0.65 . This result 
Table 5 Association of cancer-specific survival with treatment modality

\begin{tabular}{|c|c|c|c|c|c|c|}
\hline & \multicolumn{2}{|l|}{ Model 1} & \multicolumn{2}{|l|}{ Model 2} & \multicolumn{2}{|l|}{ Model 3} \\
\hline & $\mathrm{HR}(95 \% \mathrm{Cl})$ & $P$ value & $\mathrm{HR}(95 \% \mathrm{CI})$ & $P$ value & $\mathrm{HR}(95 \% \mathrm{Cl})$ & $P$ value \\
\hline \multicolumn{7}{|c|}{ Part I: univariate analysis } \\
\hline \multicolumn{7}{|l|}{ Small bowel cancer } \\
\hline None & Ref & & & & & \\
\hline Surgery only & $0.15(0.12-0.19)$ & $<0.001$ & Ref & & & \\
\hline Chemo only & $1.04(0.87-1.25)$ & 0.681 & $7.87(6.18-10.0)$ & $<0.001$ & Ref & \\
\hline Surgery + chemo & $0.37(0.29-0.47)$ & $<0.001$ & $2.55(1.93-3.38)$ & $<0.001$ & $0.32(0.25-0.41)$ & $<0.001$ \\
\hline \multicolumn{7}{|l|}{ Adenocarcinoma } \\
\hline None & Ref & & & & & \\
\hline Surgery only & $0.50(0.33-0.76)$ & 0.001 & Ref & & & \\
\hline Chemo only & $0.35(0.28-0.44)$ & $<0.001$ & $0.70(0.46-1.07)$ & 0.097 & Ref & \\
\hline Surgery + chemo & $0.24(0.18-0.33)$ & $<0.001$ & $0.47(0.29-0.75)$ & 0.002 & $0.66(0.49-0.89)$ & 0.006 \\
\hline \multicolumn{7}{|c|}{ Neuroendocrine carcinoma (NEC) } \\
\hline None & Ref & & & & & \\
\hline Surgery only & $0.25(0.18-0.35)$ & $<0.001$ & Ref & & & \\
\hline Chemo only & $0.13(0.82-1.96)$ & 0.280 & $5.20(3.35-8.07)$ & $<0.001$ & Ref & \\
\hline Surgery +chemo & $0.44(0.28-0.68)$ & $<0.001$ & $1.75(1.13-2.73)$ & 0.013 & $0.34(0.20-0.58)$ & $<0.001$ \\
\hline \multicolumn{7}{|c|}{ Gastrointestinal stromal sarcoma (GISS) } \\
\hline None & Ref & & & & & \\
\hline Surgery only & $0.51(0.14-1.94)$ & 0.324 & Ref & & & \\
\hline Chemo only & $0.18(0.05-0.68)$ & 0.012 & $0.35(0.09-1.40)$ & 0.138 & Ref & \\
\hline Surgery + chemo & $0.25(0.09-0.73)$ & 0.012 & $0.48(0.15-1.51)$ & 0.209 & $1.36(0.43-4.28)$ & 0.599 \\
\hline \multicolumn{7}{|c|}{ Part II: multivariate analysis ${ }^{\mathrm{a}}$} \\
\hline \multicolumn{7}{|l|}{ Small bowel cancer } \\
\hline None & Ref & & & & & \\
\hline Surgery only & $0.31(0.22-0.43)$ & $<0.001$ & Ref & & & \\
\hline Chemo only & $0.70(0.57-0.86)$ & 0.001 & $3.04(2.09-4.42)$ & $<0.001$ & Ref & \\
\hline Surgery + chemo & $0.41(0.30-0.57)$ & $<0.001$ & $1.48(1.07-2.04)$ & 0.018 & $0.47(0.32-0.67)$ & $<0.001$ \\
\hline \multicolumn{7}{|l|}{ Adenocarcinoma } \\
\hline None & Ref & & & & & \\
\hline Surgery only & $0.69(0.40-1.18)$ & 0.173 & Ref & & & \\
\hline Chemo only & $0.35(0.27-0.44)$ & $<0.001$ & $0.57(0.31-1.02)$ & 0.058 & Ref & \\
\hline Surgery + chemo & $0.27(0.18-0.42)$ & $<0.001$ & $0.37(0.22-0.65)$ & $<0.001$ & $0.67(0.42-1.06)$ & 0.089 \\
\hline \multicolumn{7}{|c|}{ Neuroendocrine carcinoma (NEC) } \\
\hline None & Ref & & & & & \\
\hline Surgery only & $0.24(0.14-0.43)$ & $<0.001$ & Ref & & & \\
\hline Chemo only & $1.13(0.68-1.88)$ & 0.633 & $2.81(1.41-5.63)$ & 0.003 & Ref & \\
\hline Surgery + chemo & $0.39(0.20-0.73)$ & 0.004 & $1.48(0.91-2.41)$ & 0.112 & $0.52(0.21-1.31)$ & 0.164 \\
\hline \multicolumn{7}{|c|}{ Gastrointestinal stromal sarcoma (GISS) } \\
\hline None & Ref & & & & & \\
\hline Surgery only & $0.31(0.02-4.71)$ & 0.397 & Ref & & & \\
\hline Chemo only & $0.08(0.01-0.69)$ & 0.022 & $0.34(0.04-2.81)$ & 0.314 & Ref & \\
\hline Surgery + chemo & $0.10(0.01-1.24)$ & 0.073 & $0.40(0.08-2.00)$ & 0.262 & $1.26(0.21-7.73)$ & 0.804 \\
\hline
\end{tabular}

Cl confidence interval, HR hazard ratio, Chemo chemotherapy

a Adjusted variables included age, gender, marital status, primary site, histologic grade, T stage, tumor size, $\mathrm{N}$ stage, and number of extrahepatic metastatic sites.

indicated that our combined indicators had an acceptable performance to predict the occurrence of liver metastases, and could be used to distinguish patients who need further examination, like MRI or PET-CT. 
Consistent with previous study [23, 24], our result demonstrated that patients with liver metastases had a poor survival when compared with those without liver metastases. Then, multivariate Cox analyses were performed to determine prognostic factors for small bowel cancer patients with different histological types who had liver metastases. The result showed that older age, higher histological grade, or more extrahepatic metastatic site had negative impact on prognosis of patients with NETs. In addition, small bowel cancer patients with tumors occurred in duodenum tend to have the worst prognosis both in adenocarcinoma and NETs set. However, our study found no significant prognostic factor for patients with GISTs. This result was inconsistent with previous study indicating that the prognosis of small intestinal GISTs depends upon tumors size and site of origin [25], which might owe to this subset with 92 patients only.

Due to the rarity and heterogeneity of small bowel cancer, few studies have been done to investigate the optimal treatment modalities. The management of small bowel cancer, for a long time, was based on the treatment strategy for large bowel cancer [26]. However, a number of anatomical and molecular differences strongly suggest the necessity to update the clinical management of small bowel cancer [27]. Recently, surgery is the primary therapy for most small bowel tumors presenting as locoregional disease [5, 19], which have been reported to significantly prolong patients' survival [28]. Nevertheless, for patients with metastatic small bowel cancer, the exact role of curative-intent surgery still remains unclear. In current study, the survival benefit of different treatment modalities toward patients with liver metastases was compared based on the multivariate Cox model. For adenocarcinoma, albeit no survival benefit were shown in surgical treatment alone, our result indicated that combination of surgery and systemic chemotherapy could dramatically prolonged patient's survival when compared with no treatment. In addition, consistent with previous studies $[16,29,30]$, our data also found the survival advantage of palliative chemotherapy alone. Since the high morbidity from obstruction (vomiting and poor nutrition) and bleeding caused by small bowel adenocarcinomas, it is our belief that combination of palliative surgery and systemic chemotherapy might improve prognosis and quality of life. For small bowel neuroendocrine tumors (NETs), available evidence and guidelines unanimously recommend resection of a primary tumor site and liver metastatic foci when feasible [31-34], largely because of the intermittent small bowel obstruction or even ischemia caused by neuroendocrine tumor-associated desmoplastic reaction and fibrosis [35]. Similarly, our result also demonstrated that surgery alone served as the best therapeutic option in terms of survival outcome. Furthermore, we also demonstrated that patients with gastrointestinal stromal tumors (GISTs) could only benefit from chemotherapy alone when liver metastases occurred. It was reported that adjuvant therapy of tyrosine kinase receptor inhibitor should be recommended toward those GIST patients with metastatic disease [36]. Taken together, these data showed that the optimal treatment modalities varies across different histologic types of small bowel cancer.

Inevitably, the current study has some limitations. Firstly, more detail information, such as comorbidities, performance status, the size and location of liver metastases, adjuvant therapy in terms of dose, mitotic rate, and intra-operative tumor capsule rupture, were lacking in the SEER program. Secondly, a large number of patients with incomplete or unqualified information were exclude in the study, which may induce potential selection bias. We believe that all the observed results in our study should be prospectively validated.

\section{Conclusion}

In summary, this study provided investigation of the frequency for liver metastases of small bowel cancer at initial diagnosis. Primary tumor presented with lower part of small intestine, poor tumor grade, larger tumor size, later $\mathrm{N}$ staging, and presence of more extrahepatic metastatic sites had increased propensity of developing liver metastases. The combined predictor had a good ability to predict the presence of liver metastases. Patients with liver metastases had significant poorer survival than those without liver metastases. In addition, combination of surgery and chemotherapy conferred the optimal survival for patients with adenocarcinoma, while the optimal treatment options for NEC and GISS seemed to be surgery alone and chemotherapy alone, respectively.

\section{Supplementary information}

Supplementary information accompanies this paper at https://doi. org/10.1186/s12876-020-01487-6.

Additional file 1: Table S1. Clinical characteristics of small bowel cancer patients with or without liver metastases at diagnosis.

Additional file 2: Table S2. Univariate analysis for overall survival (OS) and cancer-specific survival (CSS) among patients with small bowel adenocarcinoma who had liver metastasis.

Additional file 3: Table S3. Univariate analysis for overall survival (OS) and cancer-specific survival (CSS) among patients with small bowel neuroendocrine tumors (NETs) who had liver metastasis.

Additional file 4: Table S4. Univariate analysis for overall survival (OS) and cancer-specific survival (CSS) among patients with small bowel gastrointestinal stromal tumor (GIST) who had liver metastasis.

\section{Abbreviations}

SEER: Surveillance, Epidemiology, and End Results; NETs: Neuroendocrine tumors; GIST: Gastrointestinal stromal tumor; OS: Overall survival; CSS: Cancerspecific survival; OR: Odd ratio; HR: Hazard ratio; ROC: Receiver operating characteristic curve; AUC: Area under the curve. 


\section{Acknowledgements}

The authors acknowledged the efforts of the Surveillance, Epidemiology, and End Results (SEER) Program tumor registries in the creation of the SEER database.

\section{Authors' contributions}

$X Y, C S$ conceived and designed the present study. XY, LW, YX, and CS analyzed the data, $X Y$ interpreted the data and wrote the manuscript. All authors read and approved the final manuscript.

\section{Funding}

Not applicable.

\section{Availability of data and materials}

The datasets generated and/or analysed during the current study are available in the Surveillance, Epidemiology, and End Results Program repository, https:// seer.cancer.gov/data/.

\section{Ethics approval and consent to participate}

All analyses of human data conducted in this study were approved by the Institutional Review Board of The Lishui people's hospital and in accordance with the ethical standards of the institutional and/or national research committee and with the 1964 Helsinki declaration and its later amendments or comparable ethical standards. Informed consent was exempted because of the retrospective nature of this study. All authors signed authorization forms and received permission from SEER to access and use the dataset.

\section{Consent for publication}

Not applicable.

\section{Competing interests}

The authors declare that they have no competing interests.

Received: 23 July 2020 Accepted: 7 October 2020

Published online: 15 October 2020

\section{References}

1. Chow JS, Chen CC, Ahsan H, Neugut Al. A population-based study of the incidence of malignant small bowel tumours: SEER, 1973-1990. Int J Epidemiol. 1996;25(4):722-8.

2. Schottenfeld D, Beebe-Dimmer JL, Vigneau FD. The epidemiology and pathogenesis of neoplasia in the small intestine. Ann Epidemiol. 2009:19(1):58-69.

3. Reynolds I, Healy P, McNamara DA. Malignant tumours of the small intestine. Surg J R Coll Surg Edin Irel. 2014;12(5):263-70.

4. Cronin KA, Ries LA, Edwards BK. The Surveillance, epidemiology, and end results (SEER) Program of the National Cancer Institute. Am J Surg Pathol. 2014;120 Suppl 23:3755.

5. Dabaja BS, Suki D, Pro B, Bonnen M, Ajani J. Adenocarcinoma of the small bowel: presentation, prognostic factors, and outcome of 217 patients. Cancer. 2004;101(3):518-26

6. Manguso N, Nissen N, Hendifar A, Harit A, Amersi F. Prognostic factors influencing survival in small bowel neuroendocrine tumor with liver metastases. J Surg Oncol. 2019:18:926-31.

7. Overman MJ, Hu CY, Wolff RA, Chang GJ. Prognostic value of lymph node evaluation in small bowel adenocarcinoma: analysis of the surveillance, epidemiology, and end results database. Cancer. 2010;116(23):5374-82.

8. Minardi AJ Jr, Zibari GB, Aultman DF, McMillan RW, McDonald JC. Smallbowel tumors. J Am Coll Surg. 1998;186(6):664-8.

9. Overman MJ, Hu CY, Kopetz S, Abbruzzese JL, Wolff RA, Chang GJ. A population-based comparison of adenocarcinoma of the large and small intestine: insights into a rare disease. Ann Surg Oncol. 2012;19(5):1439-45.

10. Singhal N, Singhal D. Adjuvant chemotherapy for small intestine adenocarcinoma. Cochrane Database Syst Rev. 2007;3:Cd005202.

11. Ercolani G, Grazi GL, Ravaioli M, Ramacciato G, Cescon M, Varotti G, Del Gaudio M, Vetrone G, Pinna AD. The role of liver resections for noncolorectal, nonneuroendocrine metastases: experience with 142 observed cases. Ann Surg Oncol. 2005;12(6):459-66.

12. Adam R, Chiche $L$, Aloia T, Elias D, Salmon R, Rivoire M, Jaeck D, Saric J, Le Treut YP, Belghiti J, et al. Hepatic resection for noncolorectal nonendocrine liver metastases: analysis of 1,452 patients and development of a prognostic model. Ann Surg. 2006;244(4):524-35.

13. Howe JR, Karnell LH, Menck HR, Scott-Conner C. The American College of Surgeons Commission on Cancer and the American Cancer Society. Adenocarcinoma of the small bowel: review of the National Cancer Data Base, 1985-1995. Cancer. 1999;86(12):2693-706.

14. Jeurnink SM, van Eijck CH, Steyerberg EW, Kuipers EJ, Siersema PD. Stent versus gastrojejunostomy for the palliation of gastric outlet obstruction: a systematic review. BMC Gastroenterol. 2007;7:18.

15. Zaanan A, Costes L, Gauthier M, Malka D, Locher C, Mitry E, Tougeron D, Lecomte T, Gornet JM, Sobhani I, et al. Chemotherapy of advanced small-bowel adenocarcinoma: a multicenter AGEO study. Ann Oncol. 2010;21(9):1786-93.

16. Fishman PN, Pond GR, Moore MJ, Oza A, Burkes RL, Siu LL, Feld R, Gallinger S, Greig P, Knox JJ. Natural history and chemotherapy effectiveness for advanced adenocarcinoma of the small bowel: a retrospective review of 113 cases. Am J Clin Oncol. 2006;29(3):225-31.

17. Halfdanarson TR, MCWilliams RR, Donohue JH, Quevedo JF. A single-institution experience with 491 cases of small bowel adenocarcinoma. Am J Surg. 2010;199(6):797-803.

18. Mcnamara DA. Malignant tumours of the small intestine. Surg J R Coll Surg Edinb Irel. 2014;12(5):263-70.

19. Bilimoria KY, Bentrem DJ, Wayne JD, Ko CY, Bennett CL, Talamonti MS. Small bowel cancer in the United States: changes in epidemiology, treatment, and survival over the last 20 years. Ann Surg. 2009;249(1):63-71.

20. Sun Z, Zheng H, Yu J, Huang W, Li T, Chen H, Hu Y, Zhao M, Liu H, Jiang Y, et al. Liver metastases in newly diagnosed gastric cancer: a populationbased study from SEER. J Cancer. 2019;10(13):2991-3005.

21. Qiu MZ, Shi SM, Chen ZH, Yu HE, Sheng H, Jin Y, Wang DS, Wang FH, Li $\mathrm{YH}, \mathrm{Xie} \mathrm{D}$, et al. Frequency and clinicopathological features of metastasis to liver, lung, bone, and brain from gastric cancer: a SEER-based study. Cancer Med. 2018;7(8):3662-72

22. Smith BR, Stabile BE. Extreme aggressiveness and lethality of gastric adenocarcinoma in the very young. Arch Surg (Chicago, III: 1960). 2009;144(6):506-10

23. Wiedenmann B. Prognostic factors of long-term outcome in gastroenteropancreatic neuroendocrine tumours. Endocr Relat Cancer. 2008;15:1083-97.

24. Amersi F. Prognostic factors influencing survival in small bowel neuroendocrine tumor with liver metastases. J Surg Oncol. 2019;18:926-31.

25. Miettinen M, Lasota J. Gastrointestinal stromal tumors: pathology and prognosis at different sites. Semin Diagn Pathol. 2006;23(2):70-83.

26. Pedersen KS, Raghav K, Overman MJ. Small bowel adenocarcinoma: etiology, presentation, and molecular alterations. J Natl Compr Cancer Netw JNCCN. 2019;17(9):1135-41.

27. Benson AB, Venook AP, Al-Hawary MM, Arain MA, Chen YJ, Ciombor KK, Cohen SA, Cooper HS, Deming DA, Garrido-Laguna I, et al. Small bowel adenocarcinoma, version 1.2020, NCCN clinical practice guidelines in oncology. J Natl Compr Cancer Netw JNCCN. 2019;17(9):1109-33.

28. Raghav K, Overman MJ. Small bowel adenocarcinomas-existing evidence and evolving paradigms. Nat Rev Clin Oncol. 2013;10(9):534-44.

29. Koo DH, Yun SC, Hong YS, Ryu MH, Lee JL, Chang HM, Ryoo BY, Kang YK, Kim TW. Systemic chemotherapy for treatment of advanced small bowel adenocarcinoma with prognostic factor analysis: retrospective study. BMC Cancer. 2011;11:205.

30. Czaykowski P, Hui D. Chemotherapy in small bowel adenocarcinoma: 10-year experience of the British Columbia Cancer Agency. Clin Oncol (R Coll Radiol (Great Britain)). 2007;19(2):143-9.

31. Strosberg JR, Halfdanarson TR, Bellizzi AM, Chan JA, Dillon JS, Heaney AP, Kunz PL, O'Dorisio TM, Salem R, Segelov E, et al. The North American neuroendocrine tumor society consensus guidelines for surveillance and medical management of midgut neuroendocrine tumors. Pancreas. 2017;46(6):707-14.

32. Howe JR, Cardona K, Fraker DL, Kebebew E, Untch BR, Wang YZ, Law CH, Liu EH, Kim MK, Menda Y, et al. The surgical management of small bowel neuroendocrine tumors: consensus guidelines of the North American neuroendocrine tumor society. Pancreas. 2017:46(6):715-31. 
33. Shah MH, Goldner WS, Halfdanarson TR, Bergsland E, Berlin JD, Halperin D, Chan J, Kulke MH, Benson AB, Blaszkowsky LS, et al. NCCN guidelines insights: neuroendocrine and adrenal tumors, version 2.2018. J Natl Compr Cancer Netw JNCCN. 2018;16(6):693-702

34. Niederle B, Pape UF, Costa F, Gross D, Kelestimur F, Knigge U, Oberg K, Pavel M, Perren A, Toumpanakis C, et al. ENETS consensus guidelines update for neuroendocrine neoplasms of the jejunum and ileum. Neuroendocrinology. 2016;103(2):125-38.

35. Larouche V, Akirov A, Alshehri S, Ezzat S. Management of small bowel neuroendocrine tumors. Cancers. 2019;11(9):1935.

36. Huda T, Singh MP. Gastrointestinal stromal tumors of small intestine. Surg J (New York, NY). 2019;5(3):e92-5.

\section{Publisher's Note}

Springer Nature remains neutral with regard to jurisdictional claims in published maps and institutional affiliations.
Ready to submit your research? Choose BMC and benefit from:

- fast, convenient online submission

- thorough peer review by experienced researchers in your field

- rapid publication on acceptance

- support for research data, including large and complex data types

- gold Open Access which fosters wider collaboration and increased citations

- maximum visibility for your research: over $100 \mathrm{M}$ website views per year

At BMC, research is always in progress.

Learn more biomedcentral.com/submissions 